\title{
Tangence
}

\section{L'espace fantastique comme variété de l'espace vécu : à propos de « Stryges », nouvelle de Daniel Sernine}

\section{André Carpentier}

Numéro 50, mars 1996

Lectures de nouvelles québécoises

URI : https://id.erudit.org/iderudit/025892ar

DOI : https://doi.org/10.7202/025892ar

Aller au sommaire du numéro

Éditeur(s)

Tangence

ISSN

0226-9554 (imprimé)

1710-0305 (numérique)

Découvrir la revue

Citer cet article

Carpentier, A. (1996). L'espace fantastique comme variété de l'espace vécu : à propos de "Stryges », nouvelle de Daniel Sernine. Tangence, (50), 55-69.

https://doi.org/10.7202/025892ar d'utilisation que vous pouvez consulter en ligne.

https://apropos.erudit.org/fr/usagers/politique-dutilisation/ 


\section{L'espace fantastique comme variété de l'espace vécu : à propos de "Stryges", nouvelle de Daniel Sernine}

\section{André Carpentier}

On n'en finit jamais avec l'espace. On ne parle jamais que de lui et en lui.

\section{MiCHEL SERRES, Estbétiques sur Carpaccio}

La ville qui s'est vidée des regards humains $[\ldots]$ se contente $[\ldots]$ d'être ce silence qui appelle le sens. Elle apparait comme le lieu ultime de nos passions, de notre salut et de notre perte [...].

Pifrre Sansot, Poétique de la ville

La littérature fantastique est un lieu d'affrontements multiples. Et parmi ces affrontements, il y a ceux qui mettent l'espace en scène. Je souhaiterais ici prendre appui, au démarrage de la réflexion, sur une fiction brève relevant du genre fantastique et présentant une déambulation en milieu urbain pour essayer de dire comment les replis de la littérature fantastique de facture nouvellière nous informent sur notre rapport à cet espace urbain. Cela ne va donc pas sans postuler, comme le fait quelque part Louis Vax, que a[1]'espace fantastique est une variété de l'espace vécu " 1 .

Cette fiction, qui sera donc analysée pour des motifs qui sembleraient lui échapper, quoiqu'ils président à son exigence de forme, est de Daniel Sernine et s'intitule «Stryges *2. Qu'il soit rappelé pour information que les stryges sont des esprits nocturnes et malfaisants, des forces vampiriques qui peuvent être la métamorphose d'êtres humains vivants ou morts.

1 Louis Vax, La séduction de l'étrange, Paris, PUF., Coll. «Quadrige •, 1987 [1965], p. 196.

2 Nuits blêmes, Montréal, XYZ éditeur, coll. *L'ère nouvelle *, 1991, p. 7-19. Les références à - Stryges • renverront à cette édition. 
L'action de "Stryges" se déroule à Montréal, plus précisément dans le quartier fondateur connu sous le nom de Vieux-Montréal. Il importe de préciser que le Vieux-Montréal de Sernine renvoie à un arrondissement historique préexistant dont Sernine se sert pour développer un matériel de projection. Cet espace déambulatoire montréalais répond à la cohérence cartographique. Le décor de "Stryges" propose en effet une impression de réalité qui a pour effet de conférer au Vieux-Montréal de Sernine certaines caractéristiques du Vieux-Montréal réel, tel que compris, disons par les Montréalais ou par les touristes. Le Vieux-Montréal de Sernine propose donc une image faisant appel aux données générales et minimales d'un lieu commun, au sens, certes, d'un lieu partagé, mais aussi d'une perception conventionnelle.

Le personnage principal, qui fait ici figure d'être en détresse fantastique, se nomme Rosemonde. Curieusement, il s'agit d'un personnage au regard duquel le monde n'est justement pas rose. C'est une sans-abri, une itinérante, une clocharde. On la découvre, au début du texte, dans le restaurant du terminus d'autobus de la rue Berri, à la périphérie du Vieux-Montréal, où elle boit un café fortement arrosé d'alcool. Le tiers central de la nouvelle fait assister à la déambulation de Rosemonde entre ce terminus d'autobus et un immeuble désaffecté de la rue McGill, dàns le VieuxMontréal, qui fait office de squat ${ }^{3}$.

L'histoire de "Stryges" est la suivante. Un an plus tôt, Rosemonde a vu s'achever une aventure amoureuse peu convaincante. À une autre date qui n'est pas précisée, mais qui paraît proche de la première, elle a perdu son poste dans une société de marketing pour laquelle elle avait lancé une marque de parfum appelé Stryges. Nous sont donc ici dévoilés les motifs de son déclassement comme être social: l'amour et le travail envolés, son univers s'est écroulé. La fin de la nouvelle, dans sa partie qui suit la déambulation, présente une scène, dans l'immeuble squatté, où des stryges enlèvent et déchiquettent un jeune mendiant, fils d'une ancienne collègue de Rosemonde du temps de la promotion du parfum Stryges.

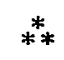

3 Immeuble désaffecté, parfois en démolition, occupé illégalement. 
Dès les premiers pas de la déambulation de Rosemonde, nous sommes informés qu'elle se rend vers le Vieux-Montréal, auquel on accède une fois * passé les stationnements enclos et les maisons de chambres, passé les chantiers et les vestiges d'incendie, passé le béton de l'autoroute et le ciment lépreux du viaduc, passé les graffiti de la détresse. (p. 9). L'aventure fantastique se développera donc dans un espace circonscrit, de l'autre côté de signes spectaculaires d'urbanité.

De fait, un certain fantastique, celui qui intéresse justement notre curiosité, exige cela : l'isolement d'un être mis en détresse au milieu de signes collectifs eux-mêmes isolés et en voie de se désincarner, des signes qui semblent vouloir retrouver leur matérialité originelle. * Stryges " présente plusieurs exemples de cet isolement. En voici quelques-uns...

Au cours d'une longue séquence - de la cinquième à la huitième page de cette fiction qui en compte treize - , Rosemonde marche en solitaire dans un espace complètement dépeuplé. Deux fois seulement elle croise une enfant porteuse d'étrange, et une fois son propre reflet dans une vitrine. Pour le reste, elle n'a autour d'elle que le construit de la cité, sans sa dimension orchestrale, sans les êtres qui font la ville vivante. Le spectacle qui est offert à Rosemonde, c'est celui d'une matrice urbaine évidée. Cet espace urbain fantastique ne charrie plus, disons à une exception près, les informations et les excitations de l'espace urbain avéré; on dirait, au lieu, une forme dont le contenu aurait été versé dans une sorte de vide limbique. Les volumes et les surfaces paraissent en repos, ce qui fait ressortir leur potentiel d'étrangeté; ce qui fait plus précisément ressortir la présence d'une dimension secrète sous la ville connue, qui ne serait pas autre chose que le squelette de la cité, sans les habitants qui la font ville. Ce fantastique me fait imaginer, à moi, une solitude plutôt impuissante, et reśsentir cette tuyauterie urbaine qui veut me digérer.

Le déambulateur usuel de nos rues guette les existences qui embarrassent l'espace urbain; dans ce fantastique qui nous intéresse, c'est plutôt le déambulateur forcé et isolé de la collectivité qui est l'objet d'une surveillance fantôme. La victime est saisie dans les conditions d'un espace rendu étrange et inquiétant: même le passage d'une enfant "menue sous un imper noir ou vert foncé" (p. 11), «à quelque distance devant elle, sur l'autre trottoir" (p. 11), fait tressaillir Rosemonde. C'est pourquoi sa marche est empreinte de gravité ( Rosemonde s'arrête à nouveau, 
58

alarmée par une onde de silence [...]. Un frisson la traverse", p. 12) en des espaces où la géométrie (marquée par des arrêts, des bifurcations, une sortie de venelle, des descentes, des "pas dont la trajectoire n'a pas été rectiligne", p. 12) et le temps qu'il fait (cette séquence de quatre pages commence par "La bruine n'a pas cessée, p. 11) ne comptent pas moins que l'état général des lieux (à l'écart de la ville animée, lieu où les vitrines poussiéreuses présentent des reflets ternes). Une catastrophe dont nous ignorons le premier mot, personnage-victime comme lecteur, paraît inévitable. En font l'annonce: du silence, de la fixité, de l'espace vide, tous éléments contraires au bruit, au mouvement et au plein rassurants de la vie quotidienne.

Là-dessus, deux remarques.

D'abord, sur la question du silence. Dès les premières pages - dès les premiers pas - de la déambulation, Rosemonde aperçoit deux fois une enfant toute menue, dans un imperméable foncé, qui traine dans les rues désertes sous la nuit bruineuse. Autrefois, apprend-on, Rosemonde aurait éprouvé de la compassion, elle aurait écrit aux journaux pour dénoncer de tels abandons d'enfants. Mais plus aujourd'hui. Elle se contente plutôt de lamper le fond de son flasque, puis de le lancer dans une allée: "aucun bruit de verre cassé" ( $p .12$ ), nous précise le texte. Comme si la victime fantastique ne pouvait plus se penser dans le monde, comme si même sa détresse ne pouvait plus faire de bruit dans la réalité.

Deuxième remarque, celle-là sur la règle de fixité et de vide. Pour confirmer cette règle par exception, il arrive qu'une population cachée se donne une présence, mais alors ce n'est que pour animer l'arrière-décor. Dans la nouvelle de Sernine, par exemple, " [l]es rues transversales offrent des aperçus de la ville, dressée par-delà la tranchée de l'autoroute, floue et fragmentée en petits carrés de lumière. Une rumeur vient de là, diffuse, le souffle de la ville. (p. 12). Tout se passe comme si le souffle fantastique, pour reprendre une expression de Jacques Finné ${ }^{4}$, exigeait une mise en marge du souffle de la ville; le souffle fantastique impose l'isolement à l'espace qui lui correspond. La rumeur urbaine qui, autrefois, conviait Rosemonde à la fréquentation, comme la veil-

4 Jacques Finné, La littérature fantastique. Essai sur longanisation sumaturelle, Bruxelles, Éditions de l'Université de Bruxelles, 1980, p. 44 sq. 
leuse attire le papillon de nuit, n'agit plus sur elle que comme une figure distante et froide. Elle n'a plus cette capacité de l'attirer de corps et d'esprit, et moi, lecteur, avec elle dans sa masse bougeante et rassurante. Cet espace "d'essence plus haute", comme dit Yves Bonnefoy ${ }^{5}$ de l'arrière-décor, Rosemonde y renonce doublement, d'abord en tant qu'être en détresse sociale, ensuite comme être en détresse fantastique. L'étrange exige la coupure d'avec le monde. En contrepartie, il y aurait peut-être lieu de postuler que l'isolement dans l'espace urbain désincarné donne accès à l'étrangeté, par reprise de contact avec ce que j'appellerais les fondements indifférents de l'espace.

Il arrive ainsi, dans la nouvelle de Sernine, comme souvent dans ce type de fantastique, que l'ordre des choses du monde immédiat manifeste son dérèglement, qu'un fragment de la ville ne rassemble plus ses signes sous une image apprivoisée, que les objets et lieux qui composent ce fragment se désincarnent et se convertissent en figures apparemment insensibles. Cet espace refait alors sa territorialité, en termes de superficie et de compétence; l'espace se confirme dans sa matière, mais se défait comme structuration à interpréter. Les signes et les repères rassurants du quotidien sont saisis et transmués par le principe moteur de l'étrangeté. Les lieux d'échange, de socialisation, de confort, les espaces de flânerie, la nature même ne parle plus son langage. Cet espace urbain, vidé d'humanité, figé dans son hypermatérialité, prend forme insécurisante, troublante. Le discours fantastique reste longtemps penché sur cette matière heurtée, soudainement privée d'humanité. C'est que la ville ne distille pas que de l'humain, elle sécrète aussi de l'inhumain, au sens de ce qui manque de ce par quoi se réaliserait pleinement la nature humaine. Les deux états se ressemblent tellement, dirait-on, ou nous sommes si aveugles, si inconscients, que nous ne voyons plus très bien la différence entre humain et inhumain. Ce à quoi le fantastique urbain donne symboliquement accès, c'est à cette

5 'J'ai souvent éprouvé un sentiment d'inquiétude, à des carrefours. Il me semble dans ces moments qu'en ce lieu ou presque; là, à deux pas sur la voie que je n'ai pas prise et dont déjà je m'éloignais, oui, c'est là que s'ouvrait un pays d'essence plus haute, où j'aurais pu aller vivre et que désormais j'ai perdu. Pourtant, tien n'indiquait ni même ne suggérait, à l'instant du choix, qu'il me fallut m'engager sur cette autre route. Yves Bonnefoy, L'arrière-pays, Paris, Flammarion, coll. *Champs (Skira-Les sentiers de la création)*, 1982 [1972], p. 7 
60

plus ou moins humaine condition. Comment justifier autrement, dans la nouvelle de Sernine, cette longue déambulation qui ne paraît pas "narrativement*-nécessaire. Postulons que sa nécessité s'est développée ailleurs, du côté de la représentation de l'espace comme variété de l'espace vécu.

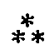

Ce qui semble donc reculer, dans le fantastique urbain, entre autres choses, c'est la pluralité de la ville qui, au mieux, n'est plus rendue que par des signes maigres. La ville vivante est ailleurs, par-delà une trancbée, floue et fragmentée. De fait, la ville fantastique retrouve une unité de principe, qui n'est rendue possible que par le déficit de population et par l'effacement des fonctions usuelles des lieux. La ville devient une masse brute désencombrée de ses croisements d'êtres. Elle prend fonction de ruine, mais sans dégradation, comme si le cours du temps s'était arrêté, et la représentation des êtres effacée. C'est par ce biais que le fantastique donne à voir une signification de l'espace qui n'est pas rassurante. L'espace fantastique, pour le personnage par exemple, est tout le contraire de jouissif, car il conjoint le pâtir à l'agir; arrivée au squat, lieu de l'épreuve fantastique, des clous agrippent son manteau, un contreplaqué se rabat sur ses doigts, "la douleur monte à son cour et elle chancelle" (p. 15), la cosse d'une plante maigre et haute l'assomme, plus loin, elle sera traversée par un spasme. C'est par cet espace et en lui que s'amorce donc l'épreuve: soit un malaise, soit un déchirement, soit une fêlure. La victime du fantastique perd l'usage de la raison spatiale; d'un étage élevé du squat, Rosemonde voit une foule de "dix mille têtes" éclairées sur l'avenue, puis ne la voit plus. Une étrangeté sinistre la guide. Dans le fantastique, l'espace urbain, comme toute chose qui vit par nous et en nous, porte le germe de sa propre destruction. La rue, la place, le carrefour, si légers et si familiers en circonstances quotidiennes, s'y appesantissent et dévoilent leur étrangeté. La matière urbaine ne semble plus touchée par l'humaine détresse. Rosemonde vacille, chancelle. Le fantastique parle beaucoup de ce refroidissement de l'espace usuel, de cette indifférence du familier qui cache, qui conduit à un piège ou à une scène mortifère.

On comprendra, dans la circonstance, que le parcours de la victime fantastique sorte du commun, non du lieu partagé, mais de 
la perception conventionnelle, et prenne tournure d'événement exceptionnel. Ce n'est pas tant la géométrie du parcours qui compte alors, mais ses propriétés spécifiques qui dessinent, dans les soubassements de la signification, une face généralement invisible de l'espace urbain. C'est ainsi que le récit prend la forme d'une poursuite sans poursuivant, d'un itinéraire sans destination, d'un maelström d'inquiétudes d'autant plus inexplicables qu'elles sont décrites à l'excès et accompagnées de pertes de vision, d'étourdissements, etc. L'espace que la victime franchit prend figure de destin. De fait, l'espace que l'on traverse est toujours un destin; dans le fantastique, il a double figure d'étrange et d'inquiétant.

\section{***}

Dans un autre ordre d'idées, on notera que Rosemonde subit une opération qui la désencombre du passé collectif; le seul passé qu'elle peut éveiller, à compter de son accès à l'espace fantastique, la concerne en propre. C'est ainsi que, quelques lignes avant son entrée dans le Vieux-Montréal, le regard porté sur un grand hôtel, celui de la Place Dupuis, avait rappelé à sa mémoire des amours déchues; et quelques lignes après son arrivée dans le quartier, la vue d'une vitrine et d'une enseigne à l'effigie de Stryges, la marque de parfum dont elle avait autrefois établi le concept, donne accès, à rebours, aux motifs de sa déchéance. Rosemonde a des souvenirs, mais des souvenirs qui ne concernent qu'elle seule et qui dévisagent son intimité. Cela a pour effet d'accentuer sa coupure d'avec le monde et de. la mettre en disposition d'accueillir l'instant surtout, disposition non seulement propice, mais nécessaire à la réception de l'étrange. Dans le fantastique, l'être en détresse est ainsi recentré sur ses émotions vives, des émotions appliquées à des faits qui ne concernent que lui seul, malgré qu'ils se déroulent au milieu de la cité.

L'être en détresse fantastique, généralement, se voit ainsi détaché des contingences du monde; Rosemonde sera, en partie d'abord, puis de plus en plus désappropriée de son caractère d'être social; elle sera poussée en exil ${ }^{6}$ dans un espace familier.

6 Curieux retournement que celui-là qui fait de l'être urbain un errant, un exilé dans la ville, justement lieu de sédentarisation des peuples nomades. 
62

Son épreuve sera d'habiter seule ces signes et de les habiter dans leur plénitude signifiante. C'est tout là le projet fantastique. Cela rappelle une idée maîtresse de la pensée de Louis Vax sur le genre fantastique: "Le sentiment de l'étrange rend l'homme étranger à lui-mêmen ${ }^{7}$. Phrase à laquelle il faudrait ajouter: ce qui, ultimement, lui permet de se rencontrer hors de toute connaissance préalable. Dans le même sens, on pourrait aussi dire que le sentiment d'étrangeté de l'espace rend l'être distant de son espace familier et le lui fait voir dans sa troublante nudité.

Par ailleurs, Rosemonde, comme être en détresse, ne déambule plus dans la ville selon sa fantaisie; elle avance plutôt mécaniquement dans un réseau d'artères, entre des façades. Les rues, les devantures sont muettes, c'est-à-dire qu'elles se condensent dans leur origine matérielle. Du coup, cependant, elles perdent leur équivoque érotique, leur motif de plaisir. L'espace urbain ravale ainsi parfois ses formes appétissantes, ses points chauds; il n'est plus un corps à caresser du regard ou du pas, mais une privation. Ce type de déambulation n'est plus vécu sur le mode de la jouissance, mais de la dépossession, de l'indigence. Plus souvent qu'autrement, la variété territoriale est effacée au profit d'un agencement plutôt uniforme et monotone.

Ce genre de fantastique, en quête d'une autre transparence de l'urbanité, renverse donc la plupart des signes urbains. C'est ce renversement qui oblige au rapport mortifère à l'urbanité. Car dans ce fantastique, l'espace n'est pas un chemin qui mène en un lieu pratique ou qui ne mène nulle part, comme dans la flânerie: cet espace fantastique ne peut que débouquer sur la perte, sur la mort - de soi, de l'autre. La marche de la victime fantastique, si elle est ici souvent ralentie, ne s'arrête jamais, jusqu'à la rencontre du foyer de l'étrange. Dans le cas de Rosemonde, on aura compris que ce sera la rencontre des stryges.

La victime fantastique parcourt donc la ville sous la menace de l'étrange, soumise à la coupure ou au renoncement à tout principe de liberté. C'est pourquoi sa mésaventure, sa détresse paraissent irréversibles. Le maléfice n'est pas qu'au bout de son trajet; il l'accompagne tout au long de sa déambulation. Il est exigé qu'avant la rencontre fatale la victime puisse vivre intensément sa perte.

7 Louis Vax, op. cit., p. 13. À noter que les éditeurs ont reporté cette phrase clé sur lá couverture de l'ouvrage. 
Au bout de cet isolement à multiples facettes, la victime en détresse fantastique n'a plus à offrir à notre regard que son état brut. Elle nous apparaît comme un être vacant, dans la même mesure que l'espace qui la porte. Rosemonde, par exemple, ne saurait même dire de quelle douleur elle souffre. Le dysfonctionnement qui l'assaille lui est insaisissable. Elle ne saurait dire non plus si ce trouble a son origine en elle ou hors d'elle. Tout ce que le discours met à la vue, c'est sa capacité à éprouver l'étrange et à pâtir. Nous assistons au spectacle de la recherche de ses limites, recherche par laquelle elle s'effectue comme être, et comme victime. C'est pourquoi l'espace fantastique apparaît souvent sous la forme d'une série de lieux piégés - ou d'un lieu à tiroirs - auxquels il est offert une résistance, certes, mais pas toujours des plus intense. Car la résistance à l'espace fantastique s'accompagne d'une charge de fascination - à moins que ce soit la fascination qui s'accompagne d'une résistance de principe.

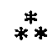

La victime fantastique est donc un être déterritorialisé. Sa lecture de la composition urbaine est froissée, chiffonnée. Dans son entendement, qui est réduit à des mécanismes simples, la cité n'est plus reçue comme une totalité ; son schéma perd l'essentiel de sa cohérence. La partie (par exemple, le Vieux-Montréal) devient oublieuse du tout. L'intelligibilité des formes urbaines est mise en doute. Ici, l'espace urbain déborde ses limites pour reprendre pied sur des origines matérielles ténébreuses. La perception visuelle s'évide de ses fondements. Les choses ne sont peut-être plus ce qu'elles paraissent être, leurs fonctions usuelles peuvent l'avoir cédé à l'organisation fantastique, c'est-à-dire à une force manipulatrice indiscernable. La syntaxe de la ville est entravée; la victime renonce à en comprendre le sens ou est impuissante à en combiner les signes. Le discours urbain familier luimême, n'offrant plus que des perspectives partielles, est soudain irrecevable comme tout articulé. La ville, produit d'une somme d'êtres historialisés, se dérobe à l'intelligence de l'un deux, qui les représente tous, en fait: qui nous représente.

Ce qui reste à cette victime plus ou moins hallucinée, c'est la contrainte de divaguer, c'est-à-dire de marcher entre des façades sans chemin fixé, tout en accomplissant un itinéraire imposé par 
64

une force insaisissable dans sa stratégie comme dans ses motifs. Ce parcours ouvre à ce qu'un espace urbain profond, caché sous les signes de surface de la ville ou du quartier, peut livrer de plus inquiétant, et que le fantastique remet en mémoire. Le fantastique prend le contre-pied de la position usuelle qui prétend que plus la ville se dévoile, mieux je la vois ${ }^{8}$. "Il se peut que la ville se dérobe davantage à mesure qu'elle se livre ", écrit Pierre Sansot ${ }^{9}$. En fait, plus elle me donne accès à son origine matérielle en se dépouillant de son humanité, moins je comprends la ville. La victime fantastique, comme ici Rosemonde, entreprend donc une errance sous forme d'un parcours imposé parce qu'elle ne comprend plus sa situation dans l'espace, parce que la signification et les valeurs de l'espace lui demeurent insaisissables. La ville manifeste, c'est-à-dire la ville humaine, se refusant, la victime affronte une matérialité froide, dépourvue de sens humain.

Alors l'être en détresse fantastique se heurte à l'autre face de la cité, à ce que j'appellerais la suburbanité, qui veut dicter sa loi de corps moral - pour la circonstance évidé de sa collectivité. L'espace urbain fantastique, c'est l'autre de la loi - opposé à l'autre du désir. Ce que le fantastique dévoile, ici, c'est l'insaisissabilité de la loi et de l'ordre qui lui correspond et qui assimile potentiellement les êtres urbains que nous sommes à une foule contrôlée.

La victime fantastique, dans la situation où elle a été mise, paraît devoir se mesurer à quelque chose qui n'est pas raccordé à son espace quotidien, quelque chose qui dépasse son humaine condition. Elle est traquée, mais elle n'a pas accès aux mobiles de cette traque. L'ordre de son monde chancelle. Les lois de la causalité s'accablent de dérèglements angoissants pour nous tous, personnages et lecteurs.

La victime fantastique, chair et sang cernés par la dureté des matériaux, est soudain pathétiquement seule; la jeune passante sous l'imperméable ne cesse de disparaître au regard de Rosemonde en se fondant dans la géométrie spatiale. Rosemonde subit ce qui n'arrive pas à tous, mais à elle seulement. L'espace urbain se transforme en traquenard personnel. On dirait une

8 On pense à Malraux disant à peu près de Balzac que plus il décrit, moins on voit.

9 Pierre Sansot, Pótique de la ville, Paris, Méridiens Klincksieck, 1984, p. 52 
grande gueule prête à l'avaler, elle, après lui avoir imposé un parcours en forme de chemin de croix. Certaines victimes pensent à la fuite - alors elles ne pensent qu'à ça; la plupart n'y pensent pas. On dirait qu'elles ne désirent plus que vivre ce qui les accable. Dans un cas comme dans l'autre, le parcours est suivi péniblement. La victime, qui ne peut ni s'arrêter ni s'évader, se fond dans la grisaille qui la fait paraître victime, en même temps que lui devient étranger le familier. Un des principes du fantastique est là, et le trouble qu'il cause: dépouiller l'être de ses attributs, l'éjecter de ses propriétés communes, et par là investir le familier d'altérité. Pour ce qui nous concerne ici, on dira que cela passe par une désincarnation - mais non par une dématérialisation - de l'espace urbain qui contamine l'être isolé qui habite cet espace. D'où l'effet fantastique.

La victime fantastique n'affiche plus aucune solidarité, elle est sans tâche dans la cité où elle ne montre plus aucune utilité; elle est animée par un innommable improductif, et dans cette improductivité, la victime n'est pas sans rien faire, elle n'est pas même au repos. Tout cela est conflictuel et indique en creux les prescriptions de la cité. Cela explique également pourquoi la victime paraît si souvent porter une culpabilité que les évênements ne justifient pas. Par ailleurs, la victime fantastique est mise hors circuit, au point d'en paraître déshumanisée à force de quête à vide d'humanité. Voilà de quoi parle le fantastique urbain; voilà en partie où se situe sa valeur imaginaire.

En fait, ce que le fantastique urbain montre, c'est que nous ne sommes pas maîtres de la signification que nous investissons dans la ville, malgré la belle assurance qui nous anime devant ce qu'elle restitue de cet investissement. Le fantastique urbain, en éloignant ce qui est proche et en rapprochant ce qui est loin dans la mémoire collective, donne à lire une image troublante de valeurs oubliées. Cela prend double forme de matière à faire peur et de portrait bougé, toujours en parcours, de cette peur.

Dans le fantastique, l'espace urbain, à la recherche de son essence, réintègre ses limites premières qui l'on fait naître ville. Le fantastique réveille et mobilise certaines évidences enfouies sous l'habitude de tels espaces. Cela a pour principal effet de contrarier la destination des espaces et objets familiers, d'en détourner le principe et de mettre en lumière la précarité humaine dans la géométrie urbaine. 
66

On sait que le fantastique canonique apparaît comme un discours poétique sur le concept d'être, sur la place, le rôle et la relation de cet être à l'univers qui le porte. Dans une perspective plus contemporaine du genre, on ajoutera: et un discours sur l'univers qu'il porte en lui. (Le lien analogique entre espace extérieur et espace intérieur n'a plus à être démontré.) Ainsi, le monde représenté, "à la fois générateur et réacteur d'incertitude et d'ambiguité", comme l'écrit Jean-Baptiste Baronian ${ }^{10}$, provoque-t-il des errements et des dérives.

Le fantastique rappelle que dans un monde où tout s'épuise vers la raison, il est une école du regard qui fait voir ce qui tient à l'insaisissable, qui se penche sur les replis traumatisant de l'être. Cette école postule un détachement brusque de la vie quotidienne pour entrer en intimité avec des terreurs sorties de forces obscures qui tiennent l'être en otage et que l'on feint d'oublier sous la matérialité mondaine. Le fantastique recherche "un ordre rompu, dit encore Baronian, un ordre essentiel que la banale réalité ne ferait jamais voir, ne cesserait pas d'occulter" ${ }^{11}$.

Le singulier, ici, est porteur d'universel. Cette vision symbolisante ouvre sur des émotions latentes, des aspects méconnus de l'humain, méconnus parce que justement trop connus, trop essentiellement perçus par des perspectives rationnelles.

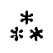

En terminant, et sans velléités conclusives, je voudrais dire qu'il est une couche fantastique, sortie du mystère de la vie collective, qui applique ses impressions d'étrangeté à l'espace quotidien, et que cette couche d'étrangeté correspond à des malaises, sans nécessairement y répondre comme un remède, des malaises liés à des émotions peu perceptibles à l'observation, mais des malaises profonds et durables: Je dirais, pour résumer, que le fantastique porte en lui tout à la fois la crainte et la curiosité d'assister au dérèglement des lois du temps et de l'espace. Le fantastique, c'est le rationalisme retourné contre lui-même: "[...] à

10 Jean-Baptiste Baronian, Un nouteau fantastique, Lausanne, L'âge d'homme, coll. " Bibliothèque fantastique *, 1977, p. 56.

11 Ibid., p. 101. 
mesure que [...] la puissance de l'homme s'est affirmée sur la machine cosmique, écrit Michel Guiomar, il lui est apparu que, malgré le degré de perfection et de rigueur du déterminisme, l'accident, l'imprévisible, l'impossible dérèglement pouvaient naître en raison même de la trop complexe harmonie des lois " 12 .

Le fantastique, en quelque sorte, pour ce qui concerne notre préoccupation d'urbanité, donne à voir des êtres en rupture de causalité avec l'espace. Cela est conforme à une définition de principe de la ville donnée par la littérature depuis la révolution industrielle, qui est comprise "comme projection des terreurs et des désirs de l'homme contemporain "13. On verra donc le fantastique comme signification interindividuelle des êtres et de l'espace. Mais aussi tout à la fois comme réduction de la capacité interprêtative de l'espace urbain et comme excès d'interprétation de la matière urbaine.

Mais qu'on me comprenne bien: le fantastique ne met le doigt sur rien qu'on puisse épeler ou lire en clair. Il suggère plutôt une percée, une avancée dans le mystère que la représentation de l'espace urbain contribue à mettre en ceuvre. C'est à chacun de nous, lecteurs de ces détresses, de mettre de la nécessité dans notre réception, de lire nos propres détresses. Plus précisément, le fantastiqueur n'a aucun message précis, préalable au texte, à communiquer. À la limite, je dirais que ce sont les lecteurs qui communiquent à travers le fantastique.

Ce type de fantastique, par lequel se rêve une détresse dans la cité, impose de repenser la corporéité dans l'espace urbain. Contre toute apparence, je dirais qu'il y a là, en creux, un effort pour rendre acceptable la condition urbaine - et par voie de conséquence, la condition humaine. Ce n'est certes pas le but profond du genre fantastique, qui n'a rien à faire de quelque forme d'axiologie que ce soit ${ }^{14}$, mais disons que le texte fantastique permet ce déploiement-là, non pas en clair, mais dans l'obscur, dans la signifiance de traces scripturaires immaîtrisables.

12 Michel Guiomar, Principes d'une estbétique de la mort, paris, José Corti, 1988, p. 277.

13 Italo Calvino, La macbine littérature, Paris, Seuil, coll. "Pierres vives", 1984, p. 47.

14 Le fantastique est ici compris comme un genre rébarbatif au message, à la leçon, à la morale... 
68

Le fantastique ne rend donc pas le réel intelligible. Il pose seulement la question de sa lisibilité. Ce qui importe, ici, ce sont les images projetées et nos terreurs assoupies qu'elles peuvent réveiller.

Le conte fantastique n'est que la croissance organique d'une impression. Le sentiment, cédant à sa pente, grossit, fait boule de neige. Mais [...] le sentiment de l'horreur a besoin d'un objet horrible qui le justifie et l'alimente. Cet objet, il le découpe d'abord dans le monde objectif. [...] la conscience horrible choisit autour d'elle ce qui justifie son horreur en écartant le reste. ${ }^{15}$

Sont donc projetées, dans le fantastique, qui est conflit du réel et du possible, des horreurs qui nous habitent. Ce ne sont pas des horreurs qui vivent seules. "Le moi et le monde ne cessent de se répondre et de s'adapter l'un à l'autre", de dire aussi $\operatorname{Vax}^{16}$.

Alors si je flirte avec le danger, comme auteur ou comme lecteur, ce serait parce que cela me met en condition d'être urbainement - inexistant; plus précisément, ça me dit que je suis proche d'être - urbainement - inexistant, et par là, cela confirme paradoxalement mon existence - urbaine... Ce retour fantastique du refoulé, c'est le prix à payer pour se savoir en vie. Comment interpréter autrement le plaisir positif que nous avons, d'abord à écrire, puis à lire ces parcours qui ont forme de sentiments négatifs? Il s'agit peut-être par là de vérifier si, en moi, la pulsion de vie s'est brisée. Si la pulsion de vie individuelle et la pulsion de vie collective se sont disloquées. Le fantastique, qui se nourrit de doutes, décidément, nous fait prendre conscience de pensées qui sont déjà en nous.

Le fantastique recherche la sensation de ce qui n'est pas advenu, mais qui, par la pensée, advient comme ressenti. Il fait vivre ce rapport à l'espace que je peux difficilement vivre consciemment parce que je le vis existentiellement.

Le fantastique participe esthétiquement d'un constat de délabrement qui ne concerne pas l'environnement seul, mais qui nous tient, lecteurs, dans une double position: subjective (nous sommes les fous de village) et objective (nous sommes critiques

15 Louis Vax, op. cit., p. 91.

16 Ibid., p. 21. 
de la vie collective: lecteurs-urbanistes, lecteurs-sociologues, lecteurs-philosophes, etc. qui nous oublions comme urbanistes, sociologues ou philosophes, parce que nous sommes, dans notre fonction de lecteur, frappés de fascination par nos propres cauchemars). Tout cela, que nous savons d'un savoir abstrait, diraiton, nous est répété dans l'obscur par le fantastique urbain, et nous rappelle, comme le dit quelque part Heidegger, qu'habiter est le trait fondamental de l'être, mais aussi, comme le suggère Michel de Certeau, qu' "on n'habite que des lieux hantés ". ${ }^{17}$.

17 Michel de Certeau, L'invention du quotidien. 1/Arts de faire, Paris, U.G.E., 10/18, coll. * Inédit *, 1980, p. 196. 